\title{
Complémentarité de la RMN, la modélisation et la diffraction pour une cristallographie des systèmes désordonnés
}

Sylvian Cadars (sylvian.cadars@cnrs-orleans.fr), Mathieu Allix, Franck Fayon, Emmanuel Véron et Dominique Massiot

CNRS, CEMHTI UPR 2079, Université d'Orléans, 45071 Orléans

Les techniques de diffraction de rayons $X$ et de neutrons, reposant sur la périodicité des réseaux cristallins, et la résonance magnétique nucléaire, qui apporte des informations sur la structure locale indépendamment de l'existence d'un ordre atomique à longue distance, sont des outils intrinsèquement complémentaires pour la cristallographie.

Nous illustrerons ici comment la combinaison de ces techniques permet d'étendre la cristallographie à des systèmes partiellement désordonnés. Dans ce contexte, la différence des échelles spatiales sondées par diffraction et RMN est un fantastique atout, en particulier lorsque ces techniques sont combinées avec la modélisation moléculaire.

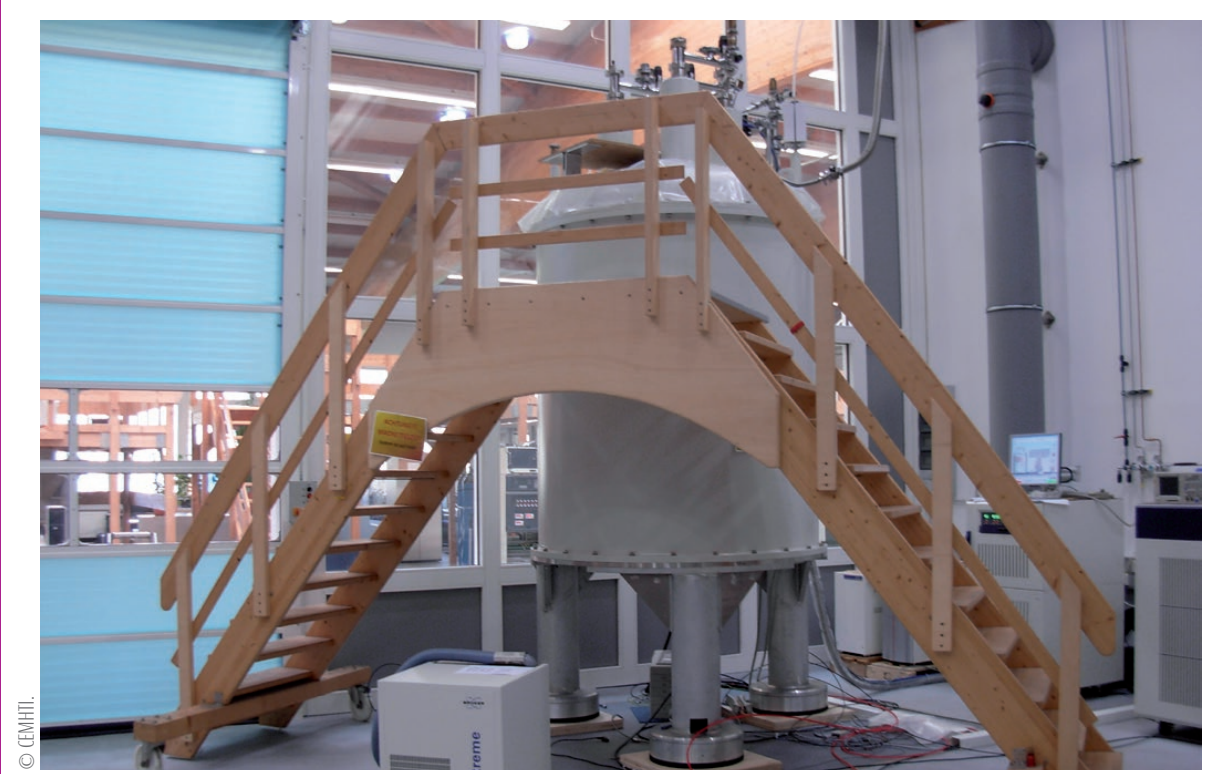

Le nouveau spectromètre RMN 850 MHZ WB du CEMHTI à Orléans, construit par Bruker et qui est arrivé en 2011. II est mis à la disposition de la communauté nationale et internationale dans le cadre de la Très Grande Infrastructure de Recherche Très Hauts Champs (TGIR RMN THC FR 3050).

La diffraction et la résonance magnétique nucléaire (RMN, voir encadré) sont des techniques de cristallographie complémentaires par nature. La première s'appuie sur la périodicité de l'arrangement atomique (la maille élémentaire) sur de grandes échelles de distance $\left(>10^{-8} \mathrm{~m}\right)$ et n'est que peu affectée par l'existence (par exemple) de défauts ponctuels. La seconde est au contraire une sonde purement locale $\left(<10^{-9} \mathrm{~m}\right)$ dans laquelle tout noyau détectable contribue de manière identique à l'intensité du signal détecté, même s'il se trouve dans un environnement désordonné, qu'il s'agisse d'un défaut local ou d'une impureté peu ou pas cristallisée. Les différentes interactions contribuant au signal RMN permettent de décrire un matériau comme un assemblage de motifs structuraux constitués d'un atome central et de ses proches voisins.

Cet article illustre à travers différents exemples cette complémentarité entre diffraction et RMN, notamment lorsqu'elles sont combinées à la chimie quantique, qui donne la possibilité de modéliser avec précision la structure atomique et électronique des matériaux, et de prédire leur réponse RMN. Elles permettent ainsi de valider des modèles structuraux, de résoudre des structures moléculaires en l'absence d'ordre atomique tridimensionnel (3D) à longue distance ou encore d'élucider les liens entre désordre structural local et propriétés des matériaux [1]. 
Principe de la résonance magnétique nucléaire à l'état solide

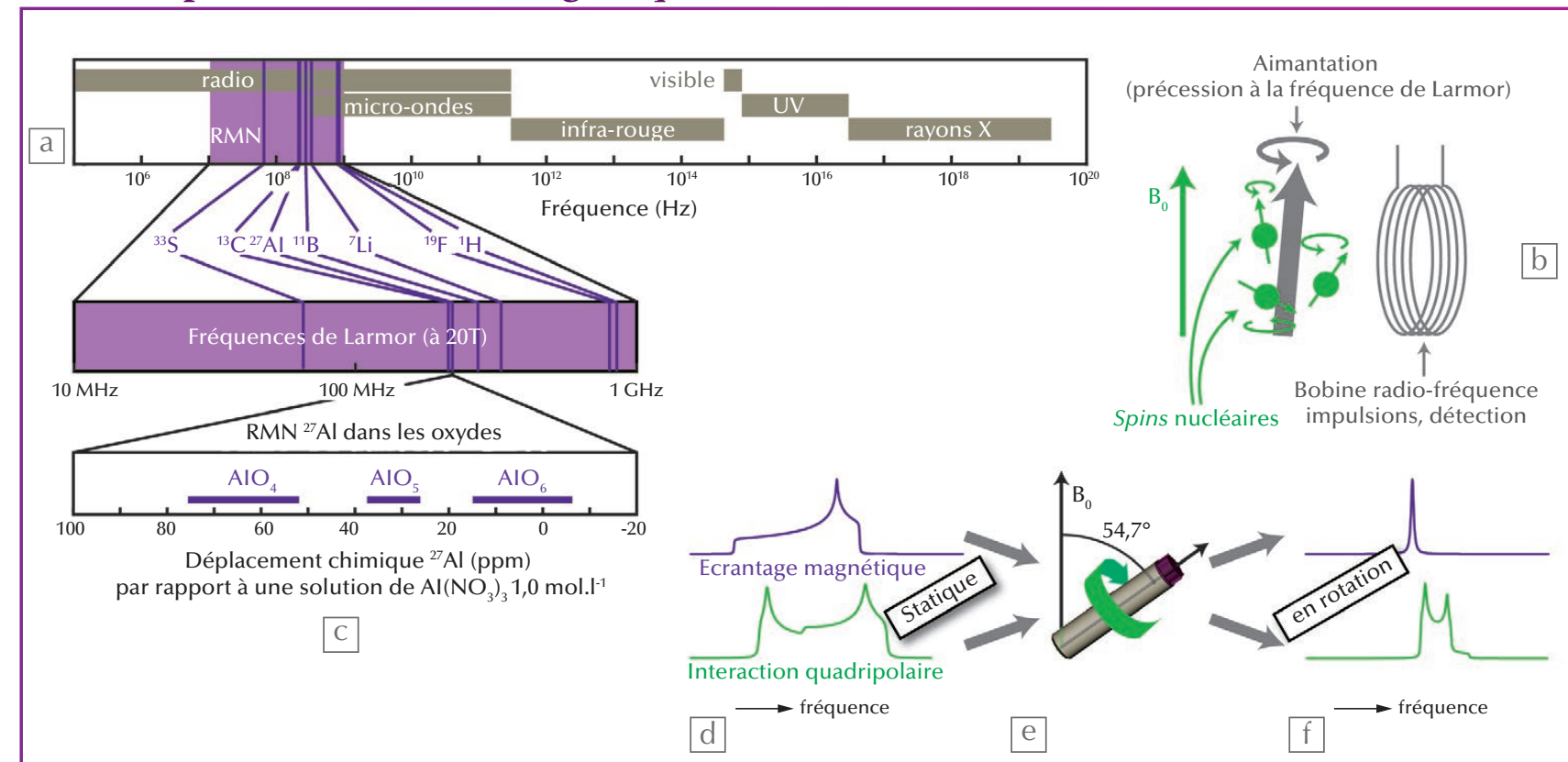

E1. Résonance magnétique nucléaire. (a) Gamme de fréquences de Larmor de quelques noyaux actifs en RMN. Elles correspondent aux fréquences de précession des moments magnétiques nucléaires soumis à un champ magnétique intense $\mathbf{B}_{\mathbf{0}}$ et de l'aimantation résultante (b). (c) Un exemple de gamme de déplacements chimiques en fonction de la structure locale : ${ }^{27} \mathrm{Al}$ dans les oxydes. (d-f) Effet de la rotation à l'angle magique (e) sur des spectres (simulés) de solides polycristallins. Les spectres de poudres dus à l'interaction de déplacement chimique (d, en violet) sont ramenés à leur composante isotrope (f, en violet), alors que l'interaction quadripolaire n'est que partiellement moyennée ( $\mathrm{d}$ et f, en vert).

La résonance magnétique nucléaire $(\mathrm{RMN})$ est une technique expérimentale qui consiste à perturber par des champs radiofréquence I'aimantation macroscopique résultant de I'alignement partiel des moments magnétiques nucléaires $\left({ }^{1} \mathrm{H},{ }^{7} \mathrm{Li},{ }^{13} \mathrm{C},{ }^{29} \mathrm{Si} . ..\right)$ placés dans un champ magnétique intense (fig. E1b). Cette spectroscopie sélective et quantitative permet d'obtenir toute une gamme d'informations sur l'environnement local des noyaux observés dans des systèmes cristallins ou amorphes, grâce aux différentes interactions qui affectent leur fréquence de résonance, et dont les effets peuvent être contrôlés au moyen d'impulsions radiofréquence.

L'identification et l'observation de manière indépendante des différents noyaux s'effectuent sur la base de leurs fréquences de Larmor (fig. E1a), les fréquences de résonance RMN qui traduisent l'éclatement des niveaux d'énergie des spins nucléaires sous l'effet du champ magnétique (effet Zeeman) et sont proportionnelles aux rapports gyromagnétiques propres à chaque isotope.

La principale sonde de la structure locale autour des noyaux est fournie par l'effet d'écrantage du champ magnétique principal par les électrons environnant le noyau observé (fig. E1c). Il est mesuré sous forme d'une interaction de déplacement chimique, exprimée en écart de fréquence par rapport à un composé de référence en parties par million (ppm) du champ magnétique, et détermine la position des pics sur le spectre.

Les proximités spatiales, voire les distances entre noyaux de même type ou différents représentent des contraintes importantes pour remonter à la structure tridimensionnelle à l'échelle locale. Elles sont mesurées à travers le couplage spin-spin direct, une interaction dipôle-dipôle qui dépend directement de la distance internucléaire.
Les connectivités chimiques, éléments centraux de l'identification de motifs structuraux, sont établies via le couplage spin-spin indirect, qui résulte du recouvrement de nuages électroniques entre atomes.

Les expériences d'édition spectrale exploitent ces couplages à travers l'espace ou les liaisons chimiques pour observer sélectivement certains noyaux en fonction de la nature chimique des atomes voisins, au moyen de jeux d'impulsions radiofréquence.

Les interactions de déplacement chimique et de couplage spin-spin dépendent de l'orientation du motif structural local par rapport au champ magnétique. Le spectre RMN d'un cristal sous forme de poudre est donc une somme pondérée des fréquences résultant de I'ensemble des orientations des cristallites, qui donne des signaux très larges et se recouvrant fortement (fig. E1d). La rotation à l'angle magique (fig. E1e) permet, dans les cas favorables, de réduire à leur composante isotrope les interactions d'écrantage (fig. E1f, en violet) et de couplage spin-spin indirect, et d'éliminer les interactions dipolaires grâce à une rotation rapide (de 10 à 100 kHz) de l'échantillon autour d'un axe formant un angle de $54.7^{\circ}$, par rapport au champ principal.

Également sensible à la structure locale, I'interaction quadripolaire mesure l'interaction entre le gradient de champ électrique local (distribution locale des charges autour du noyau considéré) et le moment quadripolaire des noyaux de spin supérieur à $1 / 2$. Dans les solides polycristallins, cette interaction donne lieu à des formes de raies RMN caractéristiques fortement dépendantes de la symétrie locale, et qui ne sont que partiellement moyennées par la rotation à I'angle magique (figs. E1d et E1f, en vert). Ces élargissements peuvent être réduits par I'utilisation de spectromètres à haut champ magnétique.

N.B. En France, I'accès à la majorité des spectromètres à très haut champ magnétique (de 17,6 à 23,5 teslas) est ouvert à l'ensemble de la communauté scientifique via un réseau national d'infrastructures de recherche (www.ir-rmn.fr). 


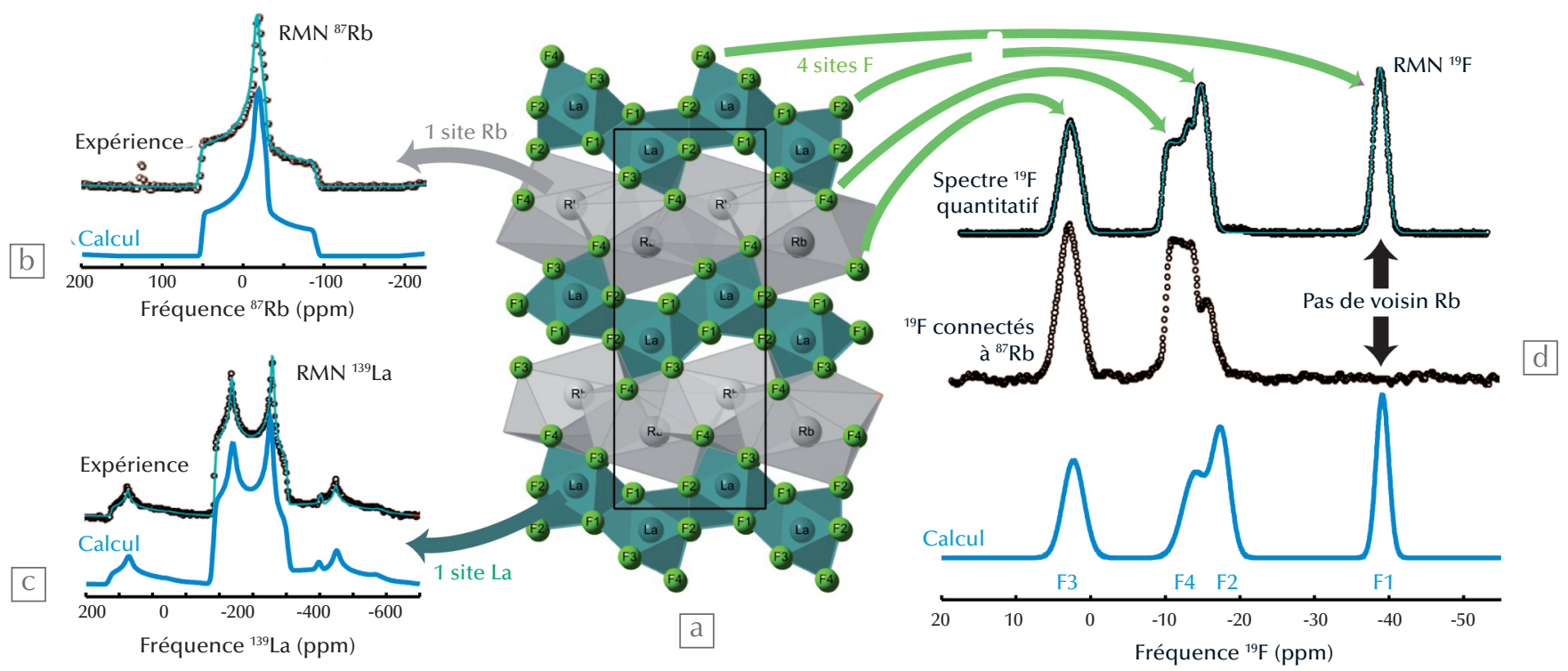

1. Validation par RMN de la structure cristalline de $\mathrm{LaRbF}_{4}$.

(a) Modèle structural issu des données de diffraction. (b, c) Spectres RMN expérimentaux (en noir) des noyaux quadripolaires ${ }^{87}$ Rb et ${ }^{139}$ La, tous deux caractéristiques d'un site unique. (d) En haut : spectre RMN expérimental du ${ }^{19} \mathrm{~F}$, qui révèle quatre sites fluor inéquivalents, en accord avec le modèle. Au milieu : spectre RMN de ${ }^{19} \mathrm{~F}$ sondant sélectivement les atomes de fluor formant une liaison chimique avec au moins un atome de rubidium, obtenu par édition spectrale (voir encadré p. 51). Sur les figures b, c et d, les spectres de RMN calculés par chimie quantique (en bleu) à partir du modèle structural (a) reproduisent parfaitement l'expérience (spectre du haut pour d), confirmant la qualité du modèle. Dans la figure $\mathrm{d}$, le bon accord entre les spectres de ${ }^{19} \mathrm{~F}$ calculé (en bas) et expérimental (en haut) fait abstraction de la forme particulière du pic expérimental à - 15 ppm, qui traduit un effet de couplage spin-spin indirect qui n'est pas pris en compte dans le calcul.

>>

\section{Validation par RMN d'une structure (sans désordre) établie par diffraction de poudre}

Une des applications les plus directes de la combinaison RMN - modélisation moléculaire en cristallographie est la validation de modèles structuraux établis à partir d'affinements de diffractogrammes.

Un tel modèle de structure a par exemple été proposé pour $\mathrm{RbLaF}_{4}$ (fig. 1a), une phase cristalline du système binaire $\mathrm{LaF}_{3}-\mathrm{RbF}$, à partir de données de diffraction de rayons $\mathrm{X}$ sur synchrotron (Argonne National Lab., USA) et des neutrons (Institut Laue-Langevin, Grenoble) [2].

La première étape est la comparaison du nombre de pics observables dans des spectres RMN quantitatifs et de leurs intensités relatives, avec le nombre de sites cristallographiques du modèle et leurs multiplicités. Les spectres RMN des isotopes ${ }^{87} \mathrm{Rb},{ }^{139} \mathrm{La}$ et ${ }^{19} \mathrm{~F}$ (fig. 1b, c et d) révèlent ainsi la présence d'un site lanthane, d'un site rubidium et de quatre sites fluor distincts de même multiplicité, en accord avec le modèle. Les formes particulières des spectres ${ }^{139} \mathrm{La}$ et ${ }^{87} \mathrm{Rb}$, qui possèdent tous deux un spin nucléaire supérieur à $1 / 2$, sont caractéristiques d'un site unique présentant une interaction quadripolaire (voir encadré p. 51).

La figure $1 \mathrm{~d}$ du milieu montre un exemple typique d'édition spectrale (voir encadré p. 51), permettant d'obtenir un spectre sélectif des noyaux de ${ }^{19} \mathrm{~F}$ liés chimiquement à des noyaux de ${ }^{87} \mathrm{Rb}$. Il révèle qu'un seul des quatre sites $\mathrm{F}$ de ce système (pic ${ }^{19} \mathrm{~F}$ à -39 ppm) n'est connecté à aucun atome de $\mathrm{Rb}$, ce qui permet d'attribuer ce signal au seul site cristallographique répondant à cette description. Les autres signaux sont attribués à partir d'expériences permettant de sonder, par exemple, les proximités spatiales entre sites $\mathrm{F}$ ou les distances La-F. L'étape finale est la comparaison entre spectres RMN expérimentaux et ceux prédits par la chimie quantique. Les spectres calculés (en bleu dans les figures $1 \mathrm{~b}-\mathrm{c}-\mathrm{d}$ ) à partir du modèle structural de $\mathrm{LaRbF}_{4}$ (fig. 1a), reproduisent avec une grande fidélité les positions de tous les pics ${ }^{19} \mathrm{~F}$ et les formes de raies quadripolaires ${ }^{139} \mathrm{La}$ et ${ }^{87} \mathrm{Rb}$. La qualité de ces prédictions démontre la parfaite validité du modèle, et atteste de l'excellente précision des positions atomiques déterminées par diffraction de rayons $\mathrm{X}$ synchrotron et de neutrons.

\section{Détermination de la structure de matériaux lamellaires sujets au désordre d'empilement}

La détermination de structures présentant un ordre atomique local, mais plus ou moins désordonnées à plus grande échelle, représente un défi majeur pour la cristallographie. C'est le cas, par exemple, des solides lamellaires utilisés comme systèmes hôtes pour l'insertion d'espèces ioniques, qu'il s'agisse d'ions $\mathrm{Li}^{+}$ pour les batteries, ou de principes actifs pour une libération contrôlée dans l'organisme.

La nature et le degré du désordre rencontrés dans ces systèmes lamellaires sont illustrés ici par une série de matériaux hybrides organique-inorganique, dont les structures ont été résolues récemment. Ces matériaux sont formés de feuillets de silicates de composition $\mathrm{Si}_{5} \mathrm{O}_{11}$, chargés négativement et séparés dans l'espace inter-feuillet par des molécules organiques cationiques qui assurent leur cohésion. Associées à des conditions de synthèse différentes, les différences de taille, de rigidité et d'affinité de ces molécules (cf. schémas moléculaires sur les figures $2 a-b-c)$ pour la surface inorganique conduisent à des degrés de cristallinité 


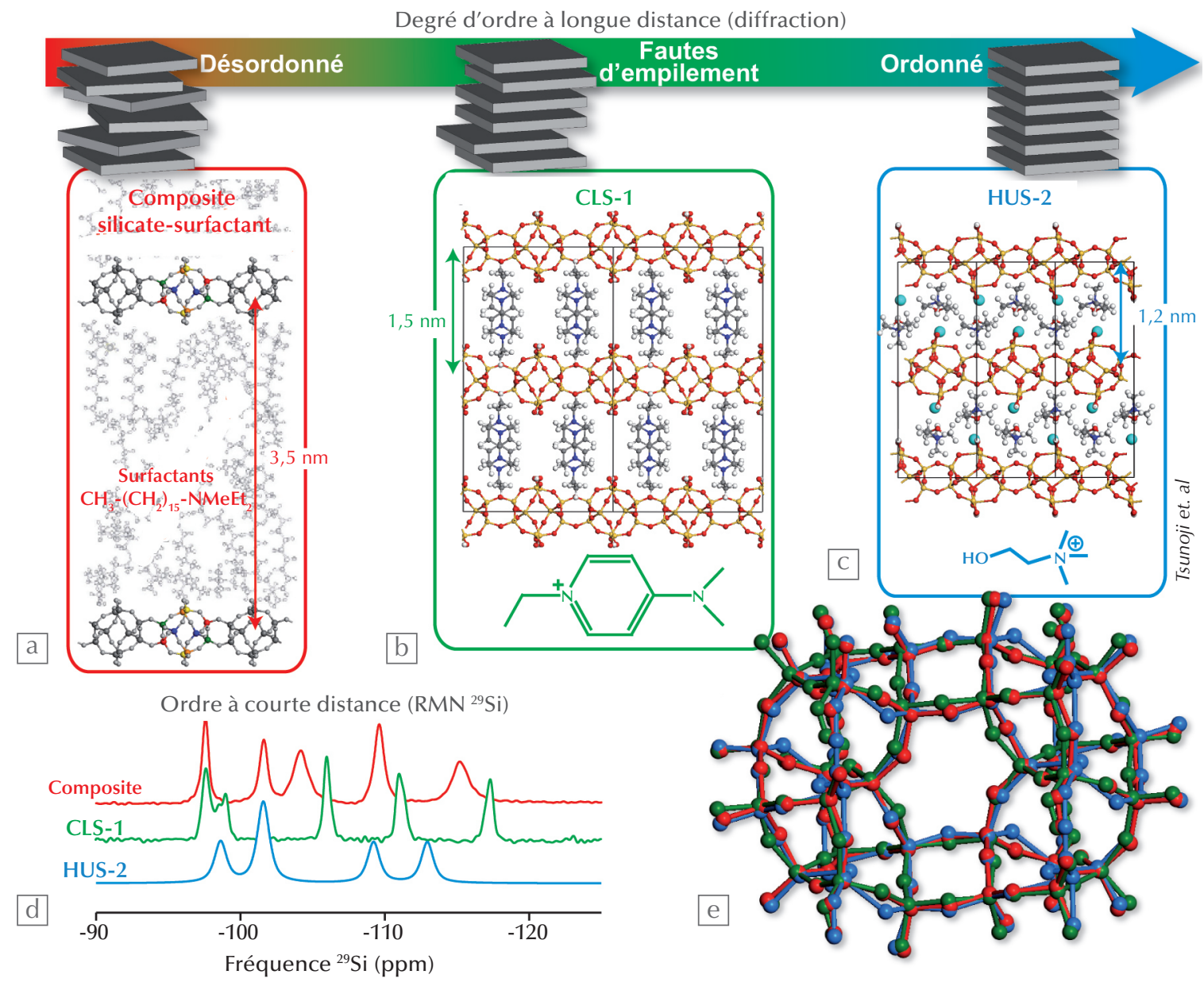

2. Composés lamellaires partiellement désordonnés.

$(a, b, c)$ Modèles structuraux de trois composés hybrides lamellaires à base de silicate, présentant des degrés très différents d'ordre d'empilement, mais tous fortement ordonnés à l'échelle du feuillet (épaisseur $<10^{-9} \mathrm{~m}$ ), comme le révèle la largeur des pics en RMN du ${ }^{29} \mathrm{Si}$ (d). Les différences de positions des pics dans les spectres RMN $\mathrm{du}{ }^{29} \mathrm{Si}$ sont imputables à des perturbations du nuage électronique des atomes de silicium par les interactions organique-inorganique. Malgré des méthodes de détermination structurale différentes (diffraction seule pour HUS-2 et à partir des données RMN pour les autres), les structures des feuillets de silicate sont globalement identiques, comme le montre leur superposition (e).
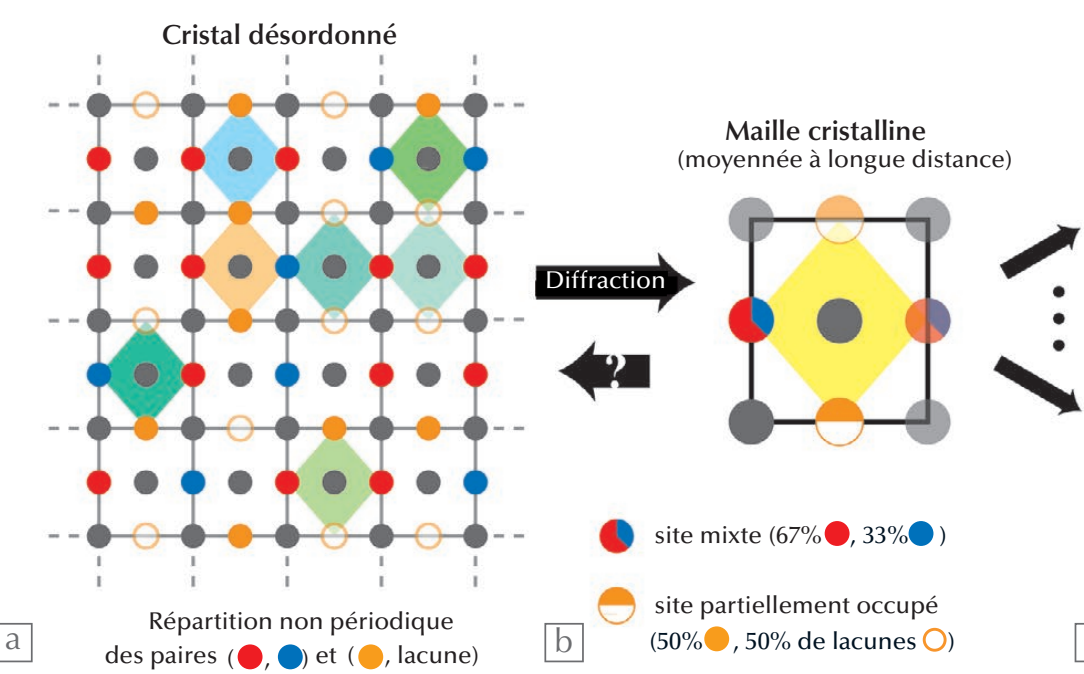

Super-cellules modèles (3×2xn mailles de départ)

3. Représentation schématique à deux dimensions d'un cristal présentant un désordre de substitution, avec un site de composition mixte (bleu/rouge) et un site partiellement occupé (orange/blanc). Contrairement au cristal réel qui contient une multitude d'environnements locaux, la maille unitaire issue de la diffraction (b) ne décrit pour chaque site qu'un environnement moyen unique. Des supercellules (c) doivent être utilisées pour modéliser les environnements locaux réels observables par RMN. 
très différents. La structure du système HUS-2 (fig. 2c, en bleu) a pu être entièrement déterminée par diffraction des rayons $\mathrm{X}$, avec un accord entre les diffractogrammes calculé et expérimental, et des formes de raies qui traduisent l'absence de fautes d'empilement [3].

Cette démarche n'est pas envisageable dans le composite silicate-surfactant (fig. 2a, en rouge), dans lequel les chaînes carbonées longues et flexibles des molécules organiques entraînent une perte de cohérence dans l'orientation des feuillets, qui élargit considérablement des pics de diffraction. Pourtant, la largeur des pics $\mathrm{RMN} \mathrm{du}{ }^{29} \mathrm{Si}$ (fig. $2 \mathrm{~d}$ ) révèle un degré d'ordre local au sein des feuillets comparable à celui de HUS-2. Cet ordre local et la faible sensibilité de la RMN au désordre d'empilement sont de formidables atouts pour déterminer la structure des feuillets, en dépit de l'absence d'ordre cristallin 3D à longue distance [4]. La RMN permet ainsi de déterminer le nombre de sites silicium distincts, leur multiplicité et leurs connectivités Si-O-Si via des atomes d'oxygène "pontants". Une analyse quantitative des proximités spatiales entre atomes de silicium via les couplages spin-spin directs (voir encadré p. 51) permet de construire des modèles de feuillets qui sont ensuite relaxés par les calculs de chimie quantique, puis discriminés sur la base de critères énergétiques et de l'accord entre paramètres RMN calculés et expérimentaux du ${ }^{29} \mathrm{Si}$. De manière spectaculaire, les modèles de feuillet retenus sont globalement identiques à celui déterminé par diffraction pour le matériau HUS-2 (fig. 2e).

La structure en feuillets du composé CLS-1 (fig. 2b, en vert) présente un degré d'ordre d'empilement intermédiaire entre les deux précédents [5]. Si, pour ce composé la diffraction n'a pas encore pu être utilisée directement pour la résolution structurale, elle permet néanmoins de sonder la nature des défauts d'empilement lorsqu'ils sont exacerbés par une augmentation de la température. Dans ce cas comme dans le précédent, la RMN du ${ }^{29} \mathrm{Si}$ (fig. 2d) est insensible à cette échelle de distance, de sorte qu'un protocole similaire a pu être mis en œuvre. Le modèle structural le plus à même de reproduire les données expérimentales de RMN donne une structure de feuillet similaire à celles des deux autres matériaux (fig. 2e). Ces exemples démontrent ainsi la possibilité d'utiliser la RMN pour étendre la

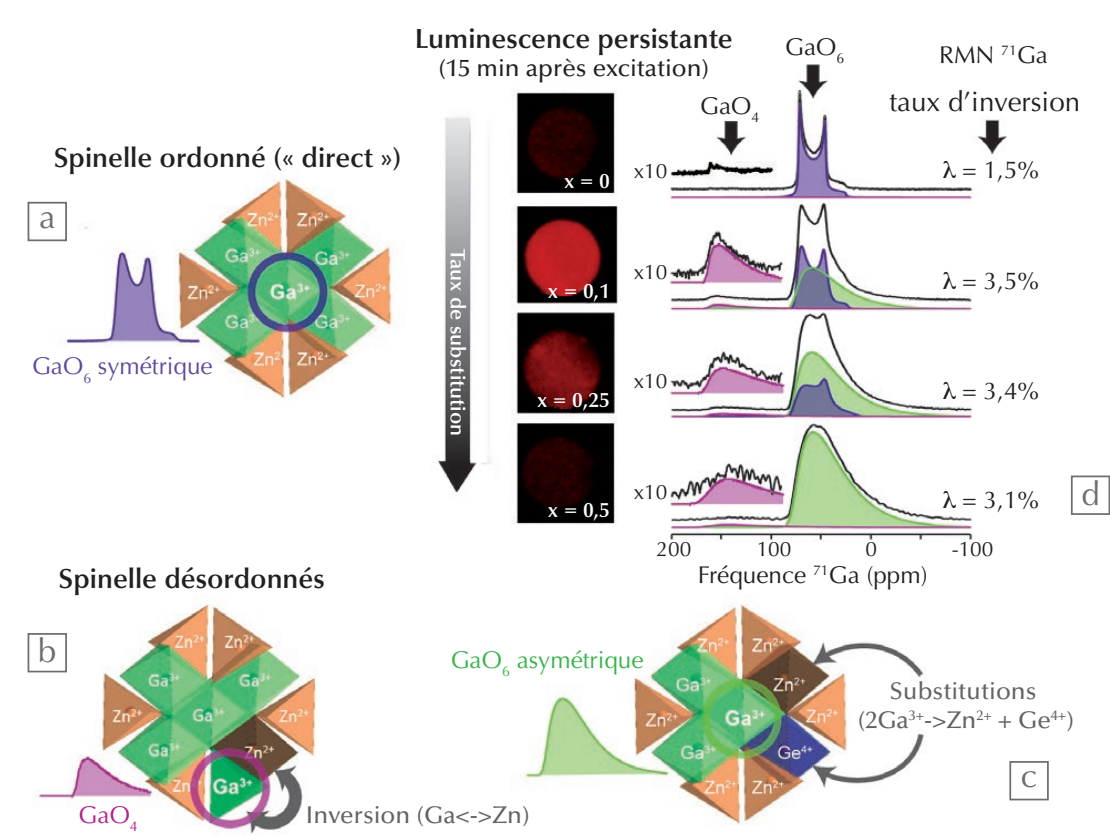

4. Propriété de luminescence persistante, et spectres RMN de l'isotope ${ }^{71} \mathrm{Ga}$, de spinelles $\mathrm{ZnGa} \mathrm{O}_{4}$ dopés avec des ions $\mathrm{Cr}^{3+}$ et substitués selon $2 \mathrm{Ga}^{3+} \rightarrow \mathrm{Zn}^{2+}+\mathrm{Ge}^{4+}$. Dans la structure spinelle directe de référence (a), les atomes de zinc occupent des tétraèdres réguliers d'oxygène (en orange) et les atomes de gallium des octaèdres réguliers d’oxygène $\mathrm{GaO}_{6}$ (entourés en bleu). (b) Le spinelle réel présente un défaut d'inversion d'occupation des sites cationiques : une petite fraction des sites tétraédriques est alors occupée par des atomes de gallium et la même quantité de sites octaédriques est occupée par les atomes de zinc, créant un premier type de défaut $\mathrm{GaO}_{4}$ (en violet) et $\mathrm{ZnO}_{6}$. (c) La substitution chimique de deux atomes de gallium par un atome de zinc et un atome de germanium génère en outre un second type de défaut d'occupation des sites octaédriques : des octaèdres $\mathrm{ZnO}_{6}$ et $\mathrm{GeO}_{6}$. L'ensemble de ces défauts d'occupation conduit à la formation de sites $\mathrm{GaO}_{6}$ irréguliers au voisinage des défauts (c, en vert). (d) La détection et la quantification des différents types de défauts et des sites octaédriques distordus au voisinage des défauts permettent de déterminer les mécanismes à l'origine de la persistance de la luminescence des dopants $\mathrm{Cr}^{3+}$.

détermination structurale au-delà des limites classiquement imposées par la diffraction seule. À partir de ces modèles de feuillets, il devient envisageable d'élargir à nouveau le point de vue, en intégrant le désordre d'empilement dans des protocoles spécialisés d'affinement des données de diffraction pour systèmes lamellaires.

\section{Le désordre de composition dans les cristaux}

La substitution atomique ou la présence de lacunes au sein de sites cristallographiques, par ailleurs bien définis, est à l'origine des propriétés d'un grand nombre de matériaux cristallins tels que les alliages métalliques, les zéolites ou les semi-conducteurs. Dans ces systèmes, la maille élémentaire déterminée par diffraction ne donne pas d'informations détaillées sur la répartition des atomes et lacunes au sein des sites mixtes ou partiellement occupés, mais fournit une vision simplifiée, correspondant à une moyenne à longue distance (fig. 3b), dans laquelle les sites désordonnés sont décrits par des probabilités d'occupation par différents atomes ou par des lacunes. Cette image simplifiée du cristal est extrêmement utile pour décrire le matériau, mais elle n'a pas de réalité physique à l'échelle locale.

Seules les spectroscopies locales, comme la RMN, peuvent sonder les différentes combinaisons d'occupation de ces sites désordonnés dans le matériau. Ces configurations multiples conduisent à un degré de complexité considérablement accru par rapport à la structure moyenne, comme illustré sur la figure 3 par les losanges de différentes couleurs. Une modélisation satisfaisante de la structure locale de tels systèmes nécessite l'emploi de super-cellules (parfois nombreuses) constituées de plusieurs mailles unitaires (fig. 3c), dans lesquelles les atomes et lacunes seront répartis au sein des sites possibles. Cette voie est aujourd'hui exploree au CEMHTI et dans d'autres laboratoires, l'enjeu consistant à identifier les combinaisons effectivement représentatives du système réel, sur la 
base de critères énergétiques ou de comparaisons entre données RMN calculées et expérimentales.

Du point de vue expérimental, la RMN fournit des informations déterminantes sur le désordre d'occupation des sites cristallographiques, pour comprendre comment ces défauts structuraux affectent les propriétés des matériaux. Dans les spinelles de composition $\mathrm{Zn}_{1+\mathrm{x}} \mathrm{Ga}_{2-2 \mathrm{x}} \mathrm{Ge}_{\mathrm{x}} \mathrm{O}_{4}$ dopés au chrome $\mathrm{Cr}^{3+}$ par exemple, la complémentarité entre RMN et diffraction permet de révéler l'origine des propriétés de luminescence persistante (fig. 4) [6]. Elles sont directement reliées à deux types de défauts par rapport à la structure de référence $(x=0)$ idéale, qui ont pu être identifiés. Le premier type de défaut est lié à une inversion de positions entre certains atomes de gallium occupant les sites octaédriques et des atomes de zinc occupant les sites tétraédriques (fig. 4b), qui peut être quantifiée (taux d'inversion $\lambda$ ) par RMN du ${ }^{71} \mathrm{Ga}$ (fig. $4 \mathrm{~d}$ ) à travers les rapports d'intensité entre sites $\mathrm{GaO}_{4}$ (en violet) et $\mathrm{GaO}_{6}$ (en vert et bleu). Dans les spinelles non substitués, ces défauts d'inversion constituent des pièges à électrons et à trous qui sont à l'origine de la luminescence retardée des ions $\mathrm{Cr}^{3+}$ occupant le site octaédrique voisin des défauts. Dans les spinelles substitués (taux de substitution $\mathrm{x}$ ), l'occupation de sites octaédriques par des atomes de germanium et de zinc constitue un second type de défaut. Dans ce cas, la mesure du degré d'inversion par RMN est nécessaire pour pouvoir déterminer les compositions moyennes $(\mathrm{Ga} / \mathrm{Zn} / \mathrm{Ge})$ de chaque site cristallographique dans la procédure d'affinement combinant les données de diffraction des rayons $\mathrm{X}$ et des neutrons. Les spectres RMN de ${ }^{71} \mathrm{Ga}$ permettent également de quantifier un second type de désordre associé à la formation de sites $\mathrm{GaO}_{6}$ distordus et désordonnés (fig. $4 c$, en vert) au voisinage des défauts de substitution, aux dépens des sites $\mathrm{GaO}_{6}$ symétriques (fig. $4 \mathrm{a}$, en bleu) présents dans la structure de référence.

Ces résultats montrent que la variation des propriétés de luminescence retardée des spinelles substitués n'est pas liée au degré d'inversion (qui reste constant), mais qu'elle résulte directement de l'occupation par les ions $\mathrm{Cr}^{3+}$ des sites $\mathrm{GaO}_{6}$ déformés par les défauts octaédriques de substitution, qui constituent des pièges électroniques profonds.

\section{Conclusion}

Reine incontestée de la cristallographie, la diffraction donne néanmoins une vision moyennée à longue distance qui peut ne refléter que partiellement la réalité de la structure à l'échelle locale. Par son caractère strictement local, la RMN est donc un outil très complémentaire susceptible de fournir des données importantes pour les protocoles de détermination ou d'affinements structuraux, telles que le nombre de sites distincts, leurs populations respectives et leurs connectivités. La comparaison de spectres RMN expérimentaux et prédits par la chimie quantique est un moyen fiable de valider et d'évaluer la qualité des modèles de structure.

Mais c'est en présence de désordre que la courte portée spatiale de la RMN devient particulièrement déterminante. Dans les systèmes en feuillets, elle la rend insensible aux fautes d'empilement qui détériorent en revanche considérablement les données de diffraction. La RMN sert alors de point de départ pour construire, affiner et valider une structure de feuillet susceptible d'être ensuite intégrée dans une analyse du désordre d'empilement basée sur la diffraction. Dans les cristaux soumis au désordre de substitution, la moyenne à longue distance de la diffraction présente l'avantage d'une grande simplicité, mais ne permet pas d'appréhender l'extrême complexité locale qui découle de ce désordre. Seul le point de vue des spectroscopies locales, dont la RMN, permet de s'y plonger, et d'espérer ainsi comprendre comment le désordre conditionne les propriétés des matériaux.

\section{Références}

1・ D. Massiot et al., Accounts of Chemical Research 46 (2013) 1975.

2 - A.-L. Rollet et al., Inorganic Chemistry 51 (2012) 2272.

3• N. Tsunoji et al., Journal of Materials Chemistry 22 (2012) 13682.

4• D.H. Brouwer et al., J. Am. Chem. Soc. 135 (2013) 5641.

5• S. Cadars et al., Chemistry of Materials 26 (2014) 6994.

6• M. Allix et al., Chemistry of Materials 25 (2013) 1600. 